\title{
Decision Support System to Analyze the Cause of Special Equipment Accident
}

\author{
JinWei SHI ${ }^{1, a}$, "Weiping OUYANG ${ }^{2, b}$, Jun QIU 2,c
}

1. Baoshan District Institute of Special Equipment Supervision and Inspeciton, Shanghai, China

2. Shanghai Institute of Special Equipment Inspection and Technical Research, Shanghai, China

bouyangweipingvi@163.com

*Weiping OUYANG

\section{Keywords: Decision Support System, Special Equipment, Complex problem}

Abstract. The development and application of Decision Support System (DSS) promote the development of related industries, special equipment accident analysis also need the support of decision support system due to variety kinds of special equipment and changeable accident cause. It was expounded definition, development situation, classification, arithmetic and existing problems of DSS according to the characteristics of special equipment accidents analysis. Research shows that there are both difference and relation among five kinds of DSS. It introduces Bayesian network and the difference of Bayesian network modeling method. The reasoning modeling method based on knowledge could use both the empirical knowledge of experts and the computer aided technology, but it is limited by the quantity and quality of expert experience knowledge similar to artificial modeling method based on expert. The application of big data, cloud computing and other related technologies in DSS would stimulate the development of DSS. It provided the technology accumulation for exploitation and application of special equipment accident analysis DSS.

\section{Introduction}

Since the concept of decision support system is put forward in 1970s, decision support system (DSS) have made remarkable progress in theoretical research, system development and practical application, which has been widely applied in machine industry, traffic, healthcare, education and so on $^{[1-7]}$. DSS could increase of the effectiveness of decisions and/or the efficiency of the decision process $^{[8]}$. The traditional decision-making mode is mainly based on the existing experience and knowledge of decision makers, which is difficult to guarantee the correctness of the decision according to the strong subjective randomness and incomplete information. The application of DSS in equipment management, equipment maintenance and emergency rescue about special equipment has been researched $^{[9-15]}$. However, failure cause of special equipment accident analysis mostly stays on the basis of the traditional decision-making mode, because the kind of special equipment is diversified, and the accident of special equipment is complex. So the cause of the accident investigation is limited by knowledge and experience of decision makers. Therefore, DSS for special equipment accident analysis is of great significance. Because of its complexity, this article mainly introduced the relevant theories of DSS, which is conducive to the establishment of DSS on Special Equipment Accident analysis.

\section{Decision Support System}

Problems according to its complexity could be subdivided into structured, semi-structured, and unstructured problems ${ }^{[15]}$. Decision-making activities could be divided into three types: structured, unstructured and semi-structured, which is consistent with problems ${ }^{[16]}$. Decision support system is the computer system which could help decision-making maker solve the semi-structured or unstructured problems. It overcomes the deficiencies of Electronic Data Processing (EDP), Management Information System (MIS) and the System Analysis (SA) ${ }^{[17]}$. It provides decision makers with a reliable solution through a series of processes of man-machine dialogue with makers, which is verified 
the requirements and ideas of makers ${ }^{[15]}$. The making process of general DSS is shown in Fig. 1. With the development of the related theory and technology, the connotation of DSS is constantly enriched and developed. However, the essence of DSS should be considered based on the system structure and its key features determined by the system ${ }^{[18]}$, which have the potential space in development and application of DSS, the key features and functions of ideal DSS are shown in Fig. 2.

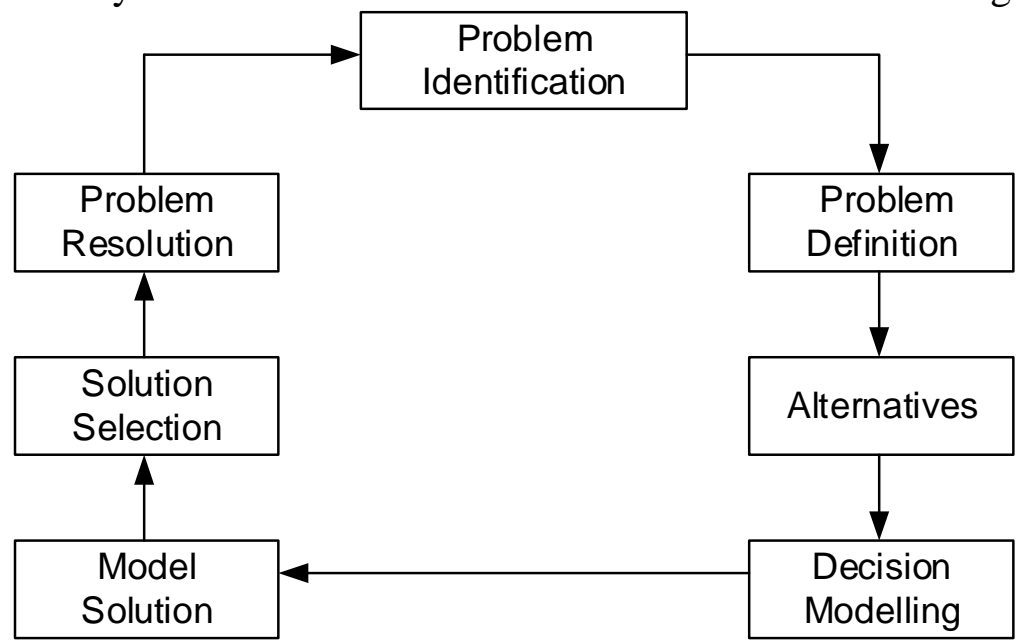

Fig. 1 The making process of general DSS

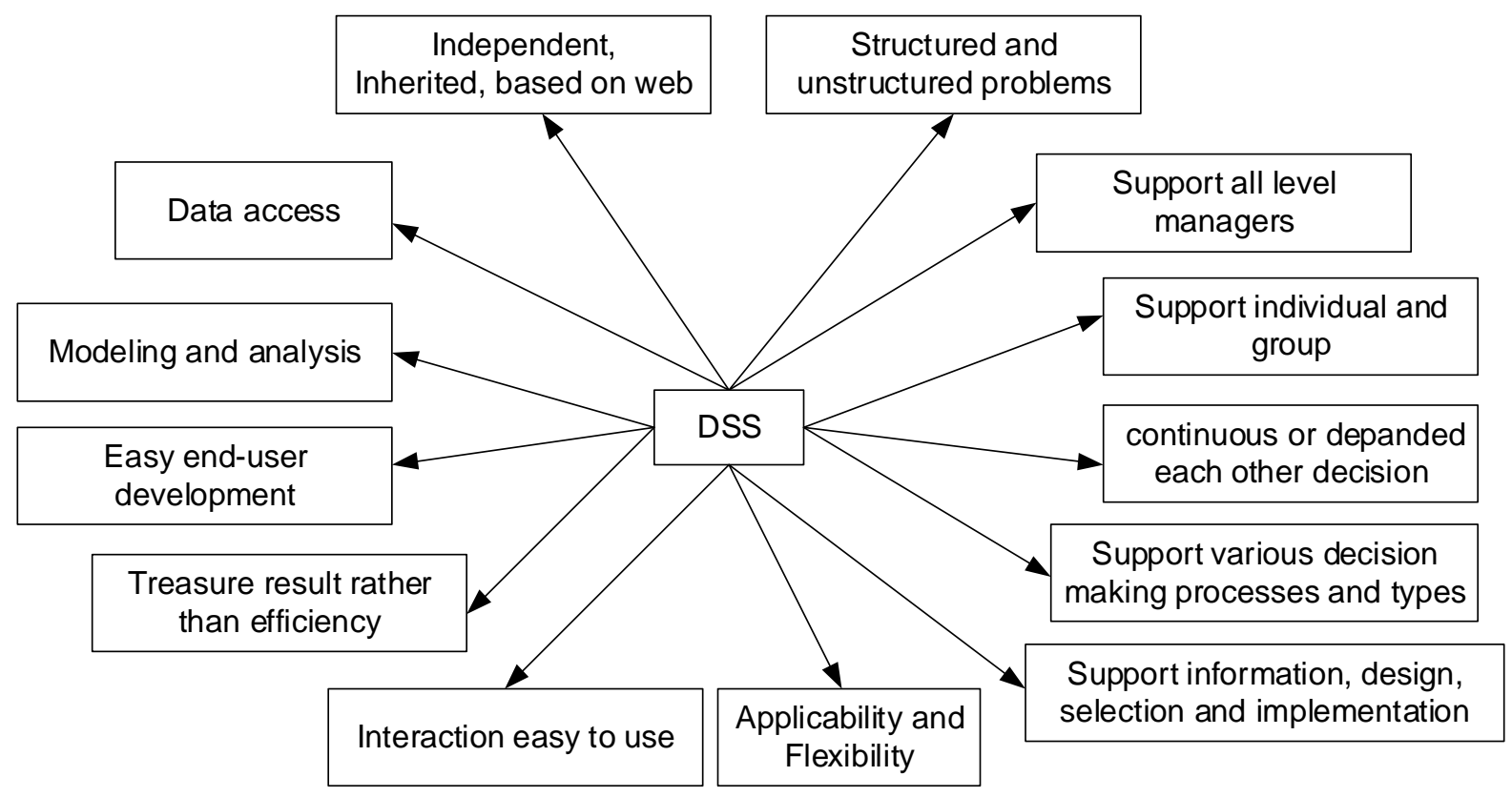

Fig. 2 The key features and functions of ideal DSS

\section{Decision Support System Framework}

Traditional DSS could be classified into two types: two databases system which is consist of dialogue component, data component and model component and three databases system which is composed of language subsystem, problem processing subsystem and knowledge subsystem. They respectively revealed internal frameworks and functional module features of DSS from different angles $^{[18]}$, which have a significant influence on the later framework development of DSS. Recently, DSS based on business intelligence technology such as Data Warehouse, On-Line Analytical Process, Data Mining and so on, has a rapid development. DSS based on database have a higher degree of standardization and product maturity than that based on model due to the substantial development and support of Microsoft, Oracle, IBM and other database vendors ${ }^{[18]}$, which becomes the main developing direction. It is generally classified by driving mode in the academia. Group Decision Supporting System 
(GDSS), Distributing Decision Supporting System (DDSS), Intelligence Decision Supporting System (IDSS), Adaptive Decision Support System (ADSS) and Intelligent, Interactive and Integrated DSS (3IDSS) are five new kinds of DSS, whose characteristics are as shown in Table 1. There are both difference and relation among five kinds of DSS. Whether GDSS or DDSS, artificial intelligence is the important way to raise the decision support capabilities. 3IDSS is the synthesis of GDSS, DDSS and ADSS essentially, which has the intelligent, interactive and integrated characteristics. It is the ideal DSS, because the user (individual, group and organization), the computer (knowledge system, expert system), the communication network, etc are reasonably used in time and space ${ }^{[20]}$. Special equipment accident analysis is a type of semi-structured problem. During the development, Intelligent DSS needs to be considered in order to achieve better decision support and adapt to the complex decision conditions.

Table 1 Characteristic of DSS

\begin{tabular}{|c|l|}
\hline Type & \multicolumn{1}{|c|}{ Characteristic } \\
\hline GDSS & $\begin{array}{l}\text { Multiple decision participants communicate ideas and information to find a } \\
\text { satisfactory and feasible solutions in system, but only a particular person } \\
\text { could make a final decision in the decision-making process, and is } \\
\text { responsible for the result of the decision }\end{array}$ \\
\hline $\begin{array}{c}\text { Distributing Decision Supporting } \\
\text { System, DDSS }\end{array}$ & $\begin{array}{l}\text { Development and application with development of Computer technology, } \\
\text { network technology and distributed database technology. System takes each } \\
\text { decision makers or organization as an independent, physically separated } \\
\text { information processing node, and provide individual support community } \\
\text { support and organizational support for these nodes. }\end{array}$ \\
\hline SupportingSystem, IDSS & $\begin{array}{l}\text { Combine Artificial Intelligence and DSS, apply Expert Systemas, make } \\
\text { DSS apply fully human knowledge or other intelligent knowledges, such as } \\
\text { descriptive knowledge related decision-making problem, procedural } \\
\text { knowledge in the process of decision-making, rational knowledge for } \\
\text { solving problem and so on. Solve the complex decision problem through } \\
\text { logical reasoning. }\end{array}$ \\
\hline Adaptive Decision Support & $\begin{array}{l}\text { In view of changeable information and the dynamic decision environment, } \\
\text { It is a dynamic, nonlinear and mutational DSS. The user could adjust } \\
\text { automaticly- or semi-automaticly system structure, function, or interface } \\
\text { according to the change of dynamic environment based on their own needs. }\end{array}$ \\
\hline System, ADSS & $\begin{array}{l}\text { Intelligent, interactive and integrated DDS are geared to the needs of } \\
\text { decision makers. Combine organically systems analysis, operations } \\
\text { research, computer technology, knowledge engineering and expert system, } \\
\text { On the premise of oriented problem, it could play their advantages, } \\
\text { especially the advantages of their combination. }\end{array}$ \\
\hline Intelligent, Interactive and Integrated \\
DSS, 3IDSS
\end{tabular}

\section{Decision Support System Arithmetic}

With the continuous development of computer and artificial intelligence technology, a variety of evaluation methods were proposed, such as bayesian network ${ }^{[22]}$, fuzzy set theory ${ }^{[23]}$, evidence theory[24] and so on. Bayesian network is based on bayesian probability theory, which is widely used in the field of artificial intelligence because of the good ability to deal with uncertainty problems. Bayesian network is modeled in form of the directed acyclic graph, the nodes represent variables in the system, the edges with the direction represents represent the causal relationship among variables, conditional probability represent the relevancy among variables, it also could be expressed and analyze the multi-source information, which helped it deal with uncertainty problems ${ }^{[25,26]}$. Both Bayesian network inference and approximate reasoning exist NP problem ${ }^{[27,28]}$, it could be reasoning efficiently in certain conditions by combining tree algorithm, Gibbs sampling algorithm, importance sampling algorithm and so on ${ }^{[29,30]}$. Bayesian network models through the following three ways: Artificial modeling method based on expert ${ }^{[31]}$, Machine learning modeling method based on sample data ${ }^{[32,33]}$ and reasoning modeling method based on knowledge ${ }^{[34]}$. Relevant fields of experts in artificial modeling method based on expert generally build artificially the structure of bayesian networks, the 
probability distribution and other related parameters. It could quickly accurately establish the topology of the bayesian network, but the certain deviation exists in determining bayesian network probabilistic distribution parameters because this method is more suitable for qualitative analysis than quantitative analysis. The core idea of Machine learning modeling method based on sample data is learning bayesian network frameworks and parameters from the sample data by a certain algorithm. The existing bayesian network machine learning method could be divided into two methods based on search score and the relation between variables, respectively ${ }^{[35]}$. The core idea of Machine learning modeling method based on sample data could established bayesian network model quantitatively, and could discover the causal relationship hidden in the sample information.

\section{Decision Support System Developments and Challenges}

There exists the following problems in existing decision support system, such as extracting knowledge difficultly, ignoring the role of people and so ${ }^{[18]}{ }^{[1}$. DSS should integrate four parts: data, models, knowledge and interaction. Numeric Calculation (such as FORTRAN, Pascal, C, etc.) is not supported for the operation of the database, and the numerical calculation ability of database language (e.g., FoxPro, Oracle, Sybase, etc.) is very weak. However, the numerical calculation and the database operations have an essential role in DSS. Otherwise, To improve the intellectualization and the decision support capabilities of DSS, some other related technologies (such as Modeling, system of rapid prototyping technology, database warehouse and data mining, knowledge discovery, knowledge, knowledge warehouse analysis technology, knowledge description and formula discovery technique, intelligent agent technique and neural network technology, visual technology, etc.) ${ }^{[21]}$. The appearance of the Internet of things, cloud computing, network supercomputers, wireless sensor, the emergence of semantic Web and other new technology promotes the development of the existing decision support system. For example, complex decision problem decision problems could be solved by cloud computing environment through the coordination of sub-problems. Firstly, complex decision problems could be decomposed into several sub problems according to certain mechanism in cloud computing environment, then the solving process carried out in different clouds. Finally, the calculation results are integrated. The development of Artificial intelligence (including genetic algorithm, fuzzy logic and the intelligent agent, etc.), Database technology, Web Service, and some special techniques in particular such as grid computing, human-computer interaction, Mobile computing and proxy heuristic search algorithm and so on provide a powerful technical support for the development of DSS and expand the depth and breadth of decision support system. There are mainly DSS development trend, including the human-computer interaction technology based on cognitive characteristics, the participation of senior people thinking, from the angle of technical and non-technical reconstruction system architecture, the integrated application of relevant technology, the intelligent system, etc.

\section{Conclusions}

In the process of decision-making theory and method development, the theory and method of intelligent decision have a great change, from single to group decision making, from the single-objective to the multi-objective decision making, from static to dynamic decisions making. As information characteristics obtained by decision-makers have changed, the decision environment has switched from certainty to uncertainty. The decision making process is from structured to unstructured. Decision support system is from centralized to distributed development accordingly. Increasingly complex and changing the environment constantly affects the description of the decision problem, the solution of making decision and the framework of DSS. Various types of special equipment and complicated and changing accident cause could make DSS for Special Equipment Accident analysis beset with difficulties. Development of cloud computing, big data and intellectualization technology provides the technical basis for application of DSS for Special Equipment Accident analysis. Special equipment accident analysis DSS could help decision maker 
accurately and quickly determine the cause of the accident, which is useful for special equipment accident prevention.

\section{References}

[1] E. B. Beşikçi, O. Arslan, O. Turan and A. I. Ölçer, Computers \& Operations Research, 66(C), (2015), p. 393.

[2] M. Colledani, O. Battaïa, CIRP Annals-Manufacturing Technology, 65(1), (2016), p. 41.

[3] Xuan Chen, Assistant Decision SuPPorting System of the Emegrence of Dangerous Cargo Conatiners in Port Area, DalianMaritimeUniversiyt. (2006). in chinese.

[4] Kai Tang, Honghong Yang and Qian Zhang, Urban Construction Theory Research, (22), (2015), p.104. in chinese.

[5] Yuhan Wang, Research on the analysis methods of road traffic accidents, Jilin University. (2011). in chinese.

[6] Bo Yu, Value Engineering, 35(34), (2016), p. 20. in chinese.

[7] Tao Liu, The Study of Machinery \& Equipment Valuation Decision Support System Based on Improvement, Hunan University. (2014). in chinese.

[8] R. Sharda, J. C. Mcdonnell, Management Science, 34(2), (1988), p. 139

[9] Liqiong Chen, Peng Zhang, Zhigang Fan and Chunlan Zhao, Oil\& Gas Storage and Transportation, 23(5), (2004), p. 19. in chinese.

[10] Wen Chen, Study on maintenance decision support system of crane based on online monitoring , Wuhan University of Technology. (2011). in chinese.

[11] Fujun Ma, Journal of Mechanical \& Electriacl Engineering, 26(9), (2009), p.104. in chinese.

[12] Xiaoying Tang, Kai Xu, Xiaolong Xue and Hongliang Lu, China Special Equipment Safety (s1), (2015), p. 12. in chinese.

[13] Xueting Wang, Design and implementation of B/S based boiler safety assistant decision-making system for thermal power plant, Shandong University. (2008). in chinese.

[14] Jian Zhang, Lei Chen and Xuedong Liang, China Special Equipment Safety, 31(3), (2015), p. 38. in chinese.

[15] Yaoye Jiao, Quality and Standardization(5), (2016), p. 47. in chinese.

[16] Gorry, G. A., and Morton, M. S. S., in: A framework for management information systems,(1971) p. 49.

[17] Guoming Yuan, Hongqi Li, Microcomputer \& Applications, 29(23), (2010), p. 5. in chinese.

[18] Xiaopeng Huang, Xianzhong Zhou, Jie Yang and Yin Sheng, Modern Management Science (4), (2012), p. 9. in chinese.

[19] Dongbo Hu, A study on model-driven decision support system, Central South University. (2009). in chinese.

[20] Qing Chen, Information Science, 23(1), (2005), p. 102. in chinese.

[21] Xinnian Wu, Yongping Chen, Information and Documentation Services (1), (2007), p. 57. in chinese.

[22] Peng, Y., Zhang, S., and Pan, R., International Journal of Uncertainty Fuzziness and Knowledge-Based Systems, 18(5), (2010), p. 539. 
[23] E. Xu , L. Fan, S. Li, J. Yang, H. Wu , T. Qu and H. Mu, 2010, Research on Preprocess Approach for Uncertain System Based on Rough Set, Springer Berlin Heidelberg.

[24] M. A. Boujelben, Y. D. Smet, A. Frikha and H. Chabchoub, International Journal of Approximate Reasoning, 52(8), (2011), p. 1171.

[25] Hänninen, M., and Kujala, P., Reliability Engineering \& System Safety, 102, (2012), p. 27.

[26] J. Stolk, in: 18th World IMACS / MODSIM Congress, Cairns, Australia , (2009), p.4339.

[27] Cooper, G. F., Probabilistic inference using belief networks is NP-hard. Knowledge Systems Laboratory, (1987).

[28] P. Dagum , M. Luby, IEEE Transactions on Pattern Analysis \& Machine Intelligence, 15(3), (1993), p. 246.

[29] H. Kozumi, G. Kobayashi, Journal of Statistical Computation \& Simulation, 81(11), (2011), p 1565 .

[30] M. S. Zaveri, H. Dan , IEEE Transactions on Nanotechnology, 9(2), (2010), p. 194.

[31] H. S. Park, S. B. Cho, , Expert Systems with Applications, 39(3), (2012), p. 2629.

[32] K. Etminani, M. Naghibzadeh and A. R. Razavi, Globally Optimal Structure Learning of Bayesian Networks from Data, Proc. Artificial Neural Networks - ICANN 2010 - International Conference, Thessaloniki, Greece, September 15-18, 2010, Proceedings, p 101.

[33] yue Wang, Shuqiu Tan and Yahui Liu, Computer Engineering, 37(7), (2011), p. 62. in chinese.

[34] Huichao Shi, Yongzheng Liang, Coal Technology, 29(9), (2010), p. 28. in chinese.

[35] D. Heckerman, Data Mining and Knowledge Discovery, 1(1), (1997), p. 79. 